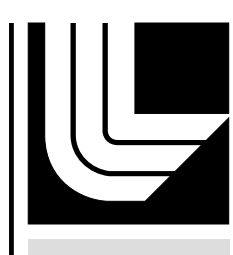

LAWRENCE LIVERMORE N A T IO N A L LABORATORY

\title{
Phenomenological Theory of the Kink Instability in a Slender Plasma Column
}

D. D. Ryutov, I. Furno, T. P. Intrator, S. Abbate, T. Madziwa-Nussinov

November 22, 2005

Physics of Plasmas 
This document was prepared as an account of work sponsored by an agency of the United States Government. Neither the United States Government nor the University of California nor any of their employees, makes any warranty, express or implied, or assumes any legal liability or responsibility for the accuracy, completeness, or usefulness of any information, apparatus, product, or process disclosed, or represents that its use would not infringe privately owned rights. Reference herein to any specific commercial product, process, or service by trade name, trademark, manufacturer, or otherwise, does not necessarily constitute or imply its endorsement, recommendation, or favoring by the United States Government or the University of California. The views and opinions of authors expressed herein do not necessarily state or reflect those of the United States Government or the University of California, and shall not be used for advertising or product endorsement purposes. 


\title{
PHENOMENOLOGICAL THEORY OF THE KINK INSTABILITY IN A SLENDER PLASMA COLUMN
}

\author{
D.D. Ryutov ${ }^{1}$, I. Furno ${ }^{2}$, T.P. Intrator ${ }^{2}$, S. Abbate ${ }^{2}$, T. Madziwa-Nussinov ${ }^{2}$ \\ ${ }^{1}$ Lawrence Livermore National Laboratory, Livermore, CA 94551 \\ ${ }^{2}$ Los Alamos National Laboratory, Los Alamos, NM 87545
}

\begin{abstract}
When one deals with a plasma column whose radius $a$ is much smaller than its length $L$, one can think of it as of a thin filament whose kink instability can be adequately described simply by a $2 \mathrm{D}$ displacement vector, $\xi_{x}=\xi_{x}(z, t) ; \xi_{y}=\xi_{y}(z, t)$. Details of the internal structure of the column such as the current, density, and axial flow velocity distribution would be lumped into some phenomenological parameters. This approach is particularly efficient in the problems with non-ideal (sheath) boundary conditions (BC) at the end electrodes, with the finite plasma resistivity, and with a substantial axial flow. With the sheath BC imposed at one of the end-plates, we find instability in the domain well below the classical Kruskal-Shafranov limit. The presence of an axial flow causes the onset of rotation of the kink and strong axial "skewness" of the eigenfunction, with the perturbation amplitude increasing in the flow direction. We consider the limitations of the phenomenological approach and find that they are related to the steepness with which the plasma resistivity increases at the plasma boundary with vacuum.
\end{abstract}

\section{INTRODUCTION}

The screw pinch is an object that has been studied for decades, in conjunction with fusion research, astrophysical problems, and basic plasma physics. One of the most salient feature of screw pinches is the development of the $m=1$ global kink mode which was a subject of numerous theoretical and experimental studies. We can refer the reader to the textbook [1] and recent publications [2-5] where further references can also be found. The role of this instability in the context of Solar physics has been discussed in a general survey [6].

In this study we suggest a simplified description of a pinch with a length $L$ much exceeding its radius $a$. What is new in our study compared to the most recent theoretical analyses of the similar problem [3,4] is that we suggest a unified way for describing such effects as boundary conditions at the electrodes (including the sheaths), finite plasma resistivity, and plasma axial flow. We call our approach "phenomenological" because it includes some ad hoc assumptions, which, although having a clear physical meaning, can be justified only in a qualitative way. This leads to the appearance of some parameters whose exact value can be found only from fitting the theory predictions and the experimental data.

Our approach is heavily based on the use of the inequality $a<L$. In this case, when becoming unstable, the $m=1$ mode causes only "gentle" wiggling of the column, so that the angle formed by the column with its unperturbed direction remains small. We do not consider here the situation where the plasma current would exceed the Kruskal- 
Shafranov current by a large margin, thereby bringing the column to the state of a violent motion and even complete disruption: we are concerned only with the states that are moderately over-critical.

We use Cartesian coordinates with the axis $z$ coinciding with the unperturbed axis of the column and the origin situated at the lower end-plate (Fig. 1). Occasionally we use also cylindrical coordinates, with the azimuthal angle $\theta$ measured from the axis $x$. We describe the deformation of the column as a result of small translation of every crosssection in the $x-y$ plane (Fig. 2), and neglect any deformation of the cross-section, so that every cross-section is merely translated in the $x-y$ plane.

One can find a justification for this assumption in that, experimentally, the fluxropes demonstrate a remarkable cohesiveness $[2,3]$. The theory justification can be found for the case where viscous and/or gyro-viscous effects [7] are important and slow down any deformations of the cross-section. The model of the "rigid" cross-sections was successfully used in the analysis of the mirror stability [8]. We do not try here to provide any deeper assessment of possible deviations from this approximation. This is yet another sign of the phenomenological nature of our approach.

Within this model the shape of the deformed column can be described by the function

$$
\boldsymbol{\xi}=\boldsymbol{\xi}(z, t)
$$

which is a two-dimensional vector of a transverse displacement of the column with respect to its unperturbed position. The condition that the deformations are "gentle" can be formulated as

$$
\left|\frac{\partial \xi}{\partial z}\right|<<1 .
$$

The description in terms of a single 2D vector-function is a key ingredient of our approach: we do not consider the inner structure of the column, only its overall shape, as determined by the displacement of circular cross-sections in the $\mathrm{x}$ and $\mathrm{y}$ directions, Fig. 2.

These assumptions mean that we cannot analyze the internal dynamics of the column, in particular, internal kinks. Our model is suitable only for considering global external kinks. The inner structure of the unperturbed column, e.g., the radial distribution of temperature and density, will be included into our model in the form of lumped phenomenological coefficients. How we implement this program, is described in the subsequent sections.

\section{THE GEOMETRY AND BASIC ORDERING}

We consider a current-carrying column of the length $L$ and radius $a$ (Fig. 1). With regard to the radius of the return current conductor $b$ we assume that it satisfies an inequality

$$
a<<b
$$

Under such conditions, $b$ drops out from the analysis [4]. [Our approach can however be easily extended to the case where $b$ becomes close to $a$, but both are considerably smaller than $L]$.

As mentioned in Introduction, we consider perturbations which can be characterized by the "rigid" displacements of the "slices" of the plasma column in the $x-y$ planes: 


$$
\xi_{x}=\xi_{x}=\xi_{x}(z), \quad \xi_{y}=\xi_{y}(z), \quad \xi_{z}=0,
$$

Fig. 2. This is a good approximation for a long-wavelength $m=1$ mode under condition (3). The axial magnetic field is large compared to the azimuthal magnetic field,

$$
B_{\varphi} / B_{z} \sim a / L<<1
$$

This corresponds to the standard scaling for the kink mode.

The plasma pressure is assumed to be small compared to the magnetic pressure, so that the axial magnetic field is almost uniform. On the other hand, the finite plasma pressure does not have any dramatic direct effect on the current-driven kinks. So, one can expect that our results will be at least qualitatively correct even at $\beta \sim 1$.

We start from the simplest model of a perfectly conducting, initially resting plasma. Later on, we add effects related to the plasma axial flow and finite plasma resistivity.

The plasma radius $a$ is defined by the condition that the conductivity outside this radius is negligibly small, and the external region can be considered as vacuum. Setting this boundary is an important step. When deforming the column in a way indicated by Eq. (4), we bend the axial magnetic field, thereby creating a restoring force. This force is the bigger the thicker bundle of field lines we bend. The bending stiffness grows with the radius $a$ of the bundle. This is reflected, in particular, in the fact that the "standard" Kruskal-Shafranov criterion $[9,10]$ for the critical current reads as:

$$
I_{k s}=\frac{\pi a^{2} B_{z} c}{L}=\frac{c \Phi}{L}
$$

where $c$ is the speed of light, and $\Phi$ is a flux of the axial magnetic field through the plasma column. We use the CGS (Gaussian) system of units. For the perturbations of the type (4) the plasma boundary is not the boundary that contains, say, $90 \%$ of the plasma mass; it is rather the boundary beyond which the conductivity becomes low. So, there is some uncertainty in the definition of $a$, and this is one of manifestations of the phenomenological nature of our model. For sharp-enough drop of the resistivity at the plasma edge this uncertainty should, however, be small. This discussion is illustrated in Fig. 3.

\section{THE CASE OF A ZERO FLOW}

\section{A. Basic equations}

The forces acting on the plasma column can be evaluated by using the energy principle. In a long-thin approximation, this has been done in a number of papers, in particular in Refs. [4] and [8]. As a result, one obtains the following equations of motion:

$$
\begin{aligned}
& \bar{\rho} \frac{\partial^{2} \xi_{x}}{\partial t^{2}}=\frac{B_{z}^{2}}{4 \pi} \frac{\partial^{2} \xi_{x}}{\partial z^{2}}-\frac{B_{z} B_{\varphi}}{4 \pi a} \frac{\partial \xi_{y}}{\partial z} \\
& \bar{\rho} \frac{\partial^{2} \xi_{y}}{\partial t^{2}}=\frac{B_{z}^{2}}{4 \pi} \frac{\partial^{2} \xi_{y}}{\partial z^{2}}+\frac{B_{z} B_{\varphi}}{4 \pi a} \frac{\partial \xi_{x}}{\partial z}
\end{aligned} .
$$

Here $B_{\varphi}$ is evaluated at the plasma boundary. The definition of the average density is:

$$
\bar{\rho}=\frac{2}{a^{2}} \int_{0}^{a} \rho r d r
$$


The first term in the right-hand-side (rhs) of Eq. (7) is related to the restoring force associated with the bending of the axial magnetic field frozen into the plasma column. [When no axial current is present, Eq. (7) describes the shear Alfven wave propagating along the column.] The second term describes an interaction of the axial current with the axial magnetic field.

By introducing a complex displacement,

$$
\eta=\xi_{x}+i \xi_{y},
$$

one can reduce set (7) to a single equation:

$$
\frac{\partial^{2} \eta}{\partial t^{2}}=\mathrm{v}_{A}^{2}\left(\frac{\partial^{2} \eta}{\partial z^{2}}+i k_{0} \frac{\partial \eta}{\partial z}\right),
$$

where

$$
k_{0}=\frac{B_{\varphi}}{a B_{z}},
$$

and $\mathrm{v}_{A} \equiv B_{z} / \sqrt{4 \pi \bar{\rho}}$ is an Alfven velocity.

For the perturbation of the form of $\exp (-i \omega t)$, one obtains:

$$
-\omega^{2} \eta=v_{A}^{2}\left(\eta^{\prime \prime}+i k_{0} \eta^{\prime}\right)
$$

where prime denotes the differentiation over $z$. Speaking of the boundary conditions for this equation, we assume that the electrode situated at $z=0$ is a perfectly conducting electrode, i.e.,

$$
\left.\eta\right|_{z=0}=0
$$

On the other hand, at this point, we do not make any assumptions about the boundary condition (BC) at $z=L$. In what follows, we implement various boundary conditions at $z=L$. It is actually not very difficult to impose an arbitrary BC at $z=0$ as well. However, this makes all the equations longer, without providing any new insights.

It is sometimes convenient to introduce a new unknown function,

$$
\tilde{\eta}=\eta \exp \left(i k_{0} z / 2\right)
$$

It satisfies the equation

$$
-\omega^{2} \tilde{\eta}=\mathrm{v}_{A}^{2}\left(\tilde{\eta}^{\prime \prime}+\frac{k_{0}^{2}}{4} \tilde{\eta}\right)
$$

with real coefficients. Multiplying this equation by $\tilde{\eta}^{*}$ (where the asterisk denotes the complex conjugate), integrating over $z$, and accounting for the boundary condition (13), one finds:

$$
\omega^{2} \int_{0}^{L}|\tilde{\eta}|^{2} d z=\mathrm{v}_{A}^{2} \int_{0}^{L}\left|\frac{\partial \tilde{\eta}}{\partial z}\right|^{2} d z-\frac{k_{0}^{2}}{4} \int_{0}^{L}|\tilde{\eta}|^{2} d z-\left.\left(\tilde{\eta} * \frac{\partial \tilde{\eta}}{\partial z}\right)\right|_{z=L}
$$

One sees that the system has a property of being self-ajoint if, at $z=L$, one of the following conditions holds: $\tilde{\eta}=0$ or $\partial \tilde{\eta} / \partial z=0$. If this is the case, the eigenfrequency is automatically either purely real (oscillations) or purely imaginary (exponential growth). In other words, in these two cases one recovers standard results of the energy principle [11]. The first boundary condition corresponds to a perfect line-tying, whereas the second one can be considered as a boundary condition on the poorly conducting surface (free sliding of the flux-tube over the end surface). We will discuss this second boundary condition in more detail in Sec III.C. 
If formulated in terms of the initial unknown function $\eta$, these boundary conditions read as:

and as

$$
\left.\eta\right|_{z=L}=0
$$

$$
\left.\left(\frac{\partial \eta}{\partial z}+\frac{i k_{0}}{2} \eta\right)\right|_{z=L}=0
$$

The solution of Eq. (12) is:

where

$$
\eta=C_{1} e^{i k_{1} z}+C_{2} e^{i k_{2} z}
$$

$$
k_{1,2}=-\frac{k_{0}}{2} \pm \sqrt{\frac{k_{0}^{2}}{4}+\frac{\omega^{2}}{\mathrm{v}_{A}^{2}}} .
$$

Imposing boundary condition of zero displacement at the surface $z=0$ (Eq. (13)), we find from Eq. (19) that $C_{1}=-C_{2}=C$, i.e.,

$$
\eta=C\left(e^{i k_{1} z}-e^{i k_{2} z}\right)
$$

where $C$ is an arbitrary normalization constant. Here we have not made any assumptions regarding the boundary condition at the other end plate.

\section{B. Line-tying at $z=L$}

For the case where there is a perfect line-tying at $z=L$, i.e., Eq. (17) holds, one has from Eq. (21):

$$
k_{1}-k_{2}=\frac{2 n \pi}{L}
$$

where $n=1,2, \ldots$ is an axial mode number. From Eqs. (20) and (22), one finds that

$$
\frac{\omega^{2}}{\mathrm{v}_{A}^{2}}=\frac{n^{2} \pi^{2}}{L^{2}}-\frac{k_{0}^{2}}{4}
$$

It is obvious that the most unstable mode corresponds to $n=1$. For this mode the critical current is equal to the Kruskal-Shafranov current $I_{K S}(6)$. Note that, at the stability boundary, the eigenfunction is $\eta=C\left(1-e^{-i k_{0} z}\right)$, i.e., it is a superposition of the helical perturbation (the second term) and a pure translation perpendicular to the pinch axis (the first term). At higher currents, the growth rate is:

$$
\operatorname{Im} \omega=\frac{\pi \mathrm{v}_{A}}{L} \sqrt{\frac{I^{2}}{I_{K S}^{2}}-1}
$$

For the normalization $C=1 / 2$ that we use throughout this paper, one has from Eq.

$$
\begin{aligned}
& \xi_{x}=\frac{1}{2}\left(\cos k_{1} z-\cos k_{2} z\right) \\
& \xi_{y}=\frac{1}{2}\left(\sin k_{1} z-\sin k_{2} z\right)
\end{aligned} .
$$

As will become clear later, this representation remains correct for a much broader set of the input parameters, e.g., for the case where the axial flow is included, or the case of the 
dissipative $\mathrm{BC}$ at the electrode $z=L$. What changes from case to case, are specific expressions for $k_{1}$ and $k_{2}$.

In the example that we are considering now, where both electrodes are perfectly conducting, we have, from Eqs. (20) and (23), with $n=1$, that

$$
k_{1}=\frac{\pi}{L}-\frac{k_{0}}{2}, k_{2}=-\frac{\pi}{L}-\frac{k_{0}}{2}
$$

From Eq. (25), we find that the perturbed axis of the column forms a helix wound over the axisymmetric surface with a radius

$$
R \equiv \sqrt{\xi_{x}^{2}+\xi_{y}^{2}}=\sin \frac{\pi z}{L}
$$

(Fig. 4). For the linear stage of the instability, this result remains valid for any currents exceeding the critical current. As $k_{1}$ and $k_{2}$ are different, the pitch of the helix, generally speaking, changes over the length. So, generally speaking, it is incorrect to describe the perturbation as a perturbation with a constant pitch.

The next axial mode, $n=2$, has a critical current equal to $2 I_{K S}$. One can therefore expect that, at $I<2 I_{K S}$, the structure of the perturbation will be defined by Eq. (25); the saturated amplitude could of course be determined only by means of the non-linear analysis (which is not a subject of this paper).

\section{Perfect sliding of the flux tube at $z=L$}

Now we consider the other "ideal" boundary condition, that described by Eq. (18). We can still use Eq. (21). When we impose boundary conditions [(13) at $z=0$ and (18) at $z=L]$, we find:

$$
\exp i\left(k_{1}-k_{2}\right) L=\frac{2 k_{2}+k_{0}}{2 k_{1}+k_{0}}
$$

Substituting $k_{1,2}$ from Eq. (20), we find

$$
\exp i\left(k_{1}-k_{2}\right) L=-1 \text {, }
$$

so that

$$
k_{1}-k_{2}=\frac{n \pi}{L}
$$

Note that, compared to Eq. (22), the r.h.s. is by a factor of 2 lower, signifying a lower instability threshold. For the most unstable mode $(n=1)$, the critical current is equal to a half of the Kruskal-Shafranov current. For higher currents, the growth-rate is:

$$
\operatorname{Im} \omega=\frac{\pi \mathrm{v}_{A}}{L} \sqrt{\frac{I^{2}}{I_{K S}^{2}}-\frac{1}{4}} .
$$

The shape of the surface on which the axis of the plasma column is wound is now

$$
R=\sin \frac{\pi z}{2 L},
$$

both for critical and super-critical current (Fig. 4b).

The critical current for the $n=2$ mode is equal to $I_{K S}$.

We should note that the boundary condition (18) is different from the boundary condition

$$
\left.\frac{\partial \eta}{\partial z}\right|_{z=L}=0
$$


that one might invoke by the analogy with the oscillation of the rod with the free end. As condition (33) is of some conceptual interest, we discuss here the dispersion relation that stems from it.

By substituting solution (21) into Eq. (33) and using Eq. (20) for $k_{1,2}$, one obtains:

$$
\tan \left(L \sqrt{\frac{k_{0}^{2}}{4}+\frac{\omega^{2}}{\mathrm{v}_{A}^{2}}}\right)=-\frac{2 i}{k_{0}} \sqrt{\frac{k_{0}^{2}}{4}+\frac{\omega^{2}}{\mathrm{v}_{A}^{2}}}
$$

This equation has an unstable solution even at small $k_{0}\left(k_{0}<<\pi / L\right)$. In the limit of small $k_{0}$, the solution of Eq. (34) is:

$$
\omega \approx \pm \frac{\pi \mathrm{v}_{A}}{L}\left(1-\frac{i k_{0} L}{2 \pi^{2}}\right)
$$

Depending on the sign of $k_{0}$, one of the roots is unstable.

\section{Sheath boundary conditions}

Thus far, we have been considering only the cases where "ideal" boundary conditions of either perfect line-tying or perfect sliding are imposed at the $z=L$ plane. In this section, we provide a qualitative phenomenological assessment of boundary conditions associated with the presence of a Debye sheath near the $z=L$ electrode.

We use the standard expression [12] for the $z$ component of the current (assuming that axial magnetic field is much greater than the azimuthal one):

$$
j_{z}=e n\left(u-\mathrm{v}_{T e} e^{-e \varphi / T_{e}}\right)
$$

Here $u$ is the ion flow velocity (of order of the ion sound speed), $n$ is the particle density, $T_{e}$ is the electron temperature, and $\varphi$ is the potential at the plasma side of the sheath with respect to the plate potential. The plate itself is considered as a good conductor, with the potential on the surface of the plate constant over the plate surface. In the unperturbed state, the current through the sheath is sustained by the externally applied voltage. Note that the role of the sheath resistance in the problems of the plasma stability has been recognized many years ago $[13,14]$.

When the magnetic field in the plasma is perturbed, then the perturbation of the $z$ component of the current is generated. By virtue of Eq. (36), this leads to the perturbation of the sheath potential. The latter means that, at the plasma side of the sheath, the tangential electric field appears, despite the fact that the underlying surface is perfectly conducting [15]. The presence of the tangential electric field in the plasma means that the lateral displacements of the plasma column become now possible, i.e., the condition of a zero lateral displacement (perfect line-tying) has to be replaced by a more general condition, which we now derive.

By perturbing Eq. (36), we find:

$$
\delta j_{z}=\alpha_{1} e n u \frac{e \delta \varphi}{T_{e}}
$$

where $\alpha_{1}$ is a numerical factor of order one:

$$
\alpha_{1}=\frac{e n u-j_{0}}{e n u},
$$

and $j_{0}$ is the unperturbed current density. We assume that $j_{0}$ does not exceed the ion saturation current. 
Axial current perturbation for the kink mode depends on the azimuth (as $\cos \theta$ ) and on the radius. Here we will not consider details of the radial dependence and use the following expression:

$$
\delta j_{z}=\alpha_{2} \frac{i c B_{z}}{4 \pi a}\left(\frac{\partial \eta}{\partial z}+i \frac{k_{0}}{2} \eta\right) .
$$

The first term in the bracket corresponds to the perturbation of the axial current caused by the bending of the axial magnetic field; the second term corresponds to the perturbation caused by the lateral displacement of the column, together with the current that it carries. Eq. (39) correctly describes the azimuthal dependence of the current perturbation, as reflected by the factors " $i$ " entering Eq. (39). More specifically, the shift of the column in the $x$ direction, creates the current perturbation with the $\cos \theta$ dependence, whereas the tilt in the $x-z$ plane creates a current perturbation with the $\sin \theta$ dependence. The coefficient in front of the bracket depends on the details of the unperturbed current distribution. So, we include a numerical form-factor $\alpha_{2}$ to account for possible uncertainty.

This discussion is qualitatively illustrated by Fig. 5 where the sheath reaction to the tilt of the column in the $x-z$ plane is shown.

By equating expressions (38) and (40), one finds that

$$
\delta \varphi=\frac{\alpha_{2}}{\alpha_{1}} \frac{c T_{e} B_{z}}{4 \pi a e^{2} n u}\left(\frac{\partial \eta}{\partial z}+i \frac{k_{0}}{2} \eta\right) .
$$

As the potential perturbation varies along the azimuth, an electric drift tangential to the surface arises, as illustrated in Fig.5 for the case where the perturbation of the column is just a tilt in the $x-z$ plane. The tangential electric field can be roughly evaluated by dividing the potential difference $\delta \varphi$ by the column radius $a$, yielding the following expression for the tangential displacement of the column imprint on the anode:

$$
\left.\eta\right|_{z=L}=\frac{c \delta \varphi}{a B_{z} \omega}
$$

or, using Eqs. (38) and (40),

$$
\eta \eta_{z=L}=-\left.\kappa \frac{i \mathrm{v}_{A}}{\omega}\left(\frac{\partial \eta}{\partial z}+i \frac{k_{0}}{2} \eta\right)\right|_{z=L}
$$

where

$$
\kappa=\frac{\alpha_{1}}{2 \alpha_{2}} \frac{c_{s}}{u}\left(\frac{c}{a \omega_{p i}}\right)^{2} \sqrt{\beta_{e}}
$$

is a dimensionless coefficient characterizing the role of the sheath resistance. The notation is:

$$
c_{s}=\sqrt{\frac{2 T_{e}}{m_{i}}} ; \beta_{e}=\frac{8 \pi n T_{e}}{B_{z}^{2}} .
$$

Again, the presence of the numerical coefficients $\alpha_{1}$ and $\alpha_{2}$ (which we cannot evaluate better than within a factor of 2 or so) points at the phenomenological nature of our model. As the order-of-magnitude estimate of the type (41) may lead to the sign error, one has to calibrate the sign from the condition that, in the case of no current, the sheath resistance should cause the damping of the standing Alfven wave. The sign presented in Eq. (42) satisfies this condition. 
We emphasize that we consider only a mode of a global displacement, where the each cross-section is simply shifted in the lateral direction (Fig. 2). In the case of smallscale perturbations, the presence of the sheath boundary condition may give rise to quite a potent instability driven mostly by the radial gradient of the electron temperature [16]. We do not consider these small-scale modes which, if present, can determine the magnitude of the anomalous transport in our problem.

Equation (42) is written in such a manner that, for the typical values of $\omega$ and $k$, $\left(\omega \sim \pi \mathrm{v}_{A} / L, k \sim \pi / L\right)$, the r.h.s. of Eq (42), aside from the coefficient $\kappa$, is of order one. Therefore, at $\kappa<<1$, one recovers the boundary condition (17) of the perfect line-tying, whereas at $\kappa \gg 1$ the boundary condition (18) becomes valid. The meaning of the parameter $\kappa$ can be understood in the following way. Consider the time of the resistive damping of the current in the circuit shown in Fig.6. The sheath resistance, according to Eq. (37), is $\sim T_{e} / \pi a^{2} e^{2} n u$, whereas the inductance of the circuit (in CGS-Gaussian system of units) is $\sim L / c^{2}$. Accordingly, the time of the resistive damping is $\sim \pi a^{2} e^{2} n u L / c^{2} T_{e}$. The ratio of the Alfven transit time $L / \mathrm{v}_{\mathrm{A}}$ to this damping time forms the parameter $\kappa \sim$ $\left(c_{s} / u\right)\left(c^{2} / a^{2} \omega_{p i}^{2}\right) \sqrt{\beta_{e}}$. For the typical parameters of the RSX experiment [3] $(a \sim 2 \mathrm{~cm}$, $\left.T_{e} \sim 10 \mathrm{eV}, n \sim 10^{13} \mathrm{~cm}^{-3}, B \sim 100 \mathrm{G}, u / c_{s} \sim 1\right)$, one has $\kappa \sim 15$.

The dispersion relation for the case of an arbitrary $\kappa$ is:

$$
\tan \left(L \sqrt{\frac{k_{0}^{2}}{4}+\frac{\omega^{2}}{\mathrm{v}_{A}^{2}}}\right)=-\frac{i \kappa \mathrm{v}_{A}}{\omega} \sqrt{\frac{k_{0}^{2}}{4}+\frac{\omega^{2}}{\mathrm{v}_{A}^{2}}}
$$

It has an aperiodic (exponentially growing) root, $\omega=i \Gamma$, with $\Gamma$ real. Introducing the dimensionless variables (denoted by a "hat") via equation

$$
\hat{k}_{0}=k_{0} L, \hat{\Gamma}=\frac{\Gamma L}{\mathrm{v}_{A}}
$$

one obtains:

$$
\tan \sqrt{\frac{\hat{k}_{0}^{2}}{4}-\hat{\Gamma}^{2}}=-\frac{\kappa}{\hat{\Gamma}} \sqrt{\frac{\hat{k}_{0}^{2}}{4}-\hat{\Gamma}^{2}},
$$

It is easy to see that the system is unstable at

$$
k_{0}>\frac{\pi}{L}
$$

(i.e., at $I>I_{K S} / 2$ ) and stable otherwise. In other words, the stability boundary is the same at any value of the parameter $\kappa$, starting from very small values corresponding to an almost perfect line-tying. However, for $\kappa<<1$ the growth rate for $k_{0}<2 \pi / L$ is small; if $\kappa<<1$, the growth rate becomes substantial only at $k_{0}>2 \pi / L$. This is illustrated by Fig. 7 .

It is also of some interest to see what happens to the dispersion relation (42) in the case corresponding to the boundary condition (33), i.e., to the assumption that the current perturbation at $z=L$ is proportional to $\partial \eta / \partial z$, without the term proportional to $k_{0} \eta$. In other words, we consider the boundary condition of the form

$$
\left.\eta\right|_{z=L}=-\left.\kappa \frac{i \mathrm{v}_{A}}{\omega} \frac{\partial \eta}{\partial z}\right|_{z=L}
$$

It leads to the dispersion relation 


$$
\tan \left(L \sqrt{\frac{k_{0}^{2}}{4}+\frac{\omega^{2}}{\mathrm{v}_{A}^{2}}}\right)=-\frac{i \kappa \mathrm{v}_{A}}{\left(\omega+\kappa \frac{\mathrm{v}_{A} k_{0}}{2}\right)} \sqrt{\frac{k_{0}^{2}}{4}+\frac{\omega^{2}}{\mathrm{v}_{A}^{2}}}
$$

At small $\kappa$ (or $k_{0}$ ) one recovers dispersion relation (45), whereas at large $\kappa$ one obtains dispersion relation (34).

\section{EFFECTS OF AN AXIAL FLOW}

We now include in our model the parallel plasma flow. We will characterize it by the velocity $\mathrm{v}$ averaged over the cross-section. The way that allows one to obtain the modified eigen-equation, is to switch to the frame where the unperturbed plasma is at rest. In that frame one recovers Eq. (10). After that, one switches back to the laboratory frame, using the Galilean transformation. This is equivalent to replacing an operator $\partial / \partial t$ by an operator $\partial / \partial \mathrm{t}+\mathrm{v} \partial / \partial \mathrm{z}$. Equation (10) is, accordingly, replaced by

$$
\bar{\rho}\left(\frac{\partial}{\partial t}+\mathrm{v} \frac{\partial}{\partial z}\right)^{2} \eta=\frac{B_{z}^{2}}{4 \pi} \frac{\partial^{2} \eta}{\partial z^{2}}+\frac{i B_{z} B_{\varphi}}{4 \pi a} \frac{\partial \eta}{\partial z} .
$$

For the perturbations of the exp-i( $\omega t-k z)$ type, one finds

$$
(\omega-k \mathrm{v})^{2}=\mathrm{v}_{A}^{2}\left(k^{2}+k_{0} k\right) \text {, }
$$

or

$$
k^{2}\left(1-M^{2}\right)+k\left(k_{0}+2 M \frac{\omega}{\mathrm{v}_{\mathrm{A}}}\right)-\frac{\omega^{2}}{\mathrm{v}_{A}^{2}}=0,
$$

where

$$
M=\frac{\mathrm{v}}{\mathrm{v}_{A}}
$$

is the Alfven Mach number. From Eq. (51), we obtain:

$$
k_{1,2}=\frac{-2 M \frac{\omega}{\mathrm{v}_{A}}-k_{0} \pm \sqrt{\left(2 M \frac{\omega}{\mathrm{v}_{A}}+k_{0}\right)^{2}+4 \frac{\omega^{2}}{\mathrm{v}_{A}^{2}}\left(1-M^{2}\right)}}{2\left(1-M^{2}\right)} .
$$

We will limit ourselves to the analysis for the two "ideal" boundary conditions (17) or (18). For the case of the perfect line-tying, substituting $k_{l, 2}$ from Eq. (53) into solution (21), and imposing the boundary condition (17), we find:

$$
\omega^{2}+\omega M k_{0} \mathrm{v}_{A}+\mathrm{v}_{A}^{2}\left[\frac{k_{0}^{2}}{4}-\left(\frac{n \pi}{L}\right)^{2}\left(1-M^{2}\right)^{2}\right]=0 .
$$

The instability criterion is:

$$
k_{0}>\frac{2 \pi}{L} \sqrt{1-M^{2}} .
$$

(we assume that $\mathrm{v}<\mathrm{v}_{\mathrm{A}}$ ). In terms of the current, the instability criterion reads:

$$
I>I_{K S} \sqrt{1-M^{2}} \text {. }
$$

The growth rate is: 


$$
\operatorname{Im} \omega=\frac{\pi \mathrm{v}_{A}}{L} \sqrt{1-M^{2}} \sqrt{\frac{I^{2}}{I_{K S}^{2}}-\left(1-M^{2}\right)}
$$

The real frequency is non-zero:

$$
\operatorname{Re} \omega=-\frac{\mathrm{v} B_{\varphi}}{2 a B_{z}}=-\frac{\pi \mathrm{v}}{L} \frac{I}{I_{K S}}
$$

As the mode under consideration is, azimuthally, the $m=1$ mode, this frequency is a rotation frequency of the perturbation. [To avoid possible misunderstanding, we emphasize that this is an "orbital" motion, in which the center of the every slice of the plasma column rotates around the $z$ axis; this is not a "proper" rotation of the unperurbed plasma.] The rotation frequency does not vary along the column length. For v positive (directed from $z=0$ to $z=L$ ) the helix screws into the $z=L$ electrode, independently of the direction of the $z$ component of the magnetic field. Note that the frequency is non-zero at the stability margin. When we go beyond the stability margin, the rotation frequency increases.

Beyond the stability margin, the axis of the plasma column is wound over the surface that is different from (27). Now it is:

$$
R=\left(\exp \frac{z \mathrm{v} \operatorname{Im} \omega}{\mathrm{v}_{A}^{2}-\mathrm{v}^{2}}\right)\left(\sin \frac{\pi z}{L}\right)
$$

Note that this surface does not possess a symmetry with respect to the $L / 2$ plane. The perturbation is "skewed" in the direction of flow (Fig. 4 a, dashed line).

Consider now the stability margin in the case of the boundary condition (18), i.e., $\kappa \rightarrow \infty$. An inspection of Eq. (28) shows that the stability boundary corresponds to

$$
k_{0}=\frac{\pi}{L} \sqrt{1-M^{2}} \text {, }
$$

or

$$
\frac{I_{c r i t}}{I_{K S}}=\frac{\sqrt{1-M^{2}}}{2} .
$$

The parameters $k_{1}$ and $k_{2}$ that enter the eigenfunction (21) are (at the stability boundary):

$$
k_{1}=\frac{k_{0}}{2}\left(\frac{1}{\sqrt{1-M^{2}}}-1\right) ; k_{2}=-\frac{k_{0}}{2}\left(\frac{1}{\sqrt{1-M^{2}}}+1\right),
$$

The rotation frequency of the mode is:

$$
\omega=-\frac{\pi \mathrm{v}}{L} \sqrt{1-M^{2}}
$$

with the helix screwing into the $z=L$ electrode. The surface over which the axis of the perturbed plasma column is wound is still the same as (32).

We do not present here a lengthy analysis of Eq. (28) for super-critical currents and just note that, in the supercritical domain with the flow present, the surface (32) gets skewed in the direction of the flow, as sketched in Fig. 4b, dashed line.

\section{THE EFFECTS OF BULK RESISTIVITY}

Now we switch to the effects of the bulk plasma resistivity. Here one has to realize that, in the unperturbed state, the steady plasma current is sustained by the applied 
voltage. When the column starts to wiggle, the voltage is still on, and the current is still sustained. Conversely, the bending of the axial field lines is not supported by the applied voltage and, at a finite plasma resistivity, the field begins to slip against the plasma. If one bends the column very slowly, the axial magnetic field is left behind, and loses its stabilizing effect.

This can be expressed in a more quantitative way by the following equation describing bending of the axial magnetic field by the perturbations:

$$
\delta \boldsymbol{B}=i k B_{z} \xi-\frac{i \delta \boldsymbol{B}}{(\omega-k \mathrm{v}) \tau_{r}} .
$$

Here we introduced the resistive time $\tau_{r}$ for a phenomenological characterization of the resistive dissipation of the perturbations of the axial field:

$$
\tau_{r}=\frac{2 \pi a^{2} \sigma}{c^{2}}
$$

where $\sigma$ is the electrical conductivity of the plasma. Note that we do not solve the problem of the magnetic field diffusion, just introduce a characteristic time. One can rewrite Eq. (64) as:

$$
\delta \boldsymbol{B}=\frac{i(\omega-k \mathrm{v}) \tau_{r}}{1+i(\omega-k \mathrm{v}) \tau_{r}} i k B_{z} \xi .
$$

When $\tau_{r}$ is infinite, we recover the result of the perfect line-tying. Conversely, when the diffusion time is short, the bending of the axial field becomes very weak $(\delta \mathbf{B}$ vanishes). In this case, the critical current reduces to zero.

According to this discussion, Eq. (50) has now to be modified:

$$
(\omega-k \mathrm{v})^{2}=\mathrm{v}_{A}^{2}\left[\frac{i(\omega-k \mathrm{v}) \tau_{r}}{1+i(\omega-k \mathrm{v}) \tau_{r}}\left(k^{2}+\frac{k_{0} k}{2}\right)+\frac{k_{0} k}{2}\right]
$$

Note that, for a nonzero flow velocity, the equation for $k$ becomes of the third order, thereby requiring the imposition of one more boundary condition. This would be the condition that $\delta \boldsymbol{B}$ is zero at $z=0$ (the finite-conductivity fluid cannot carry a current sheath, and enters the volume in question without any initial perturbation of the magnetic field). Implementing this boundary condition in the general case is quite an onerous task. The boundary condition at $z=L$ also becomes quite complex.

The dimensionless parameter that characterizes the role of resistive effects is

$$
\zeta=\frac{\pi \mathrm{v}_{A} \tau_{r}}{L}
$$

(this is a product of the characteristic frequency and the resistive time). For the parameters of the RSX experiment, $\left(L \sim 100 \mathrm{~cm}, a \sim 2 \mathrm{~cm}, T_{e} \sim 10 \mathrm{eV}, n \sim 10^{13} \mathrm{~cm}^{-3}, B \sim 100\right.$ $\mathrm{G})$, one has $\xi \sim 6$.

The main role of the resistive effects is related to the decrease of the critical current. Equation (69) below shows that slow-enough perturbations in a non-flowing plasma would become unstable even at the current substantially lower than the KS current. In other words, if resistive modes are considered, a "hard" stability limit is replaced by a gradual emergence of the slow modes, which begin to show up at relatively low currents. The addition of the flow, however, leads to at least partial stabilization of these modes. All these effects are encapsulated in relatively simple (algebraic!) equations of our phenomenological model. 
To illustrate the effect of a finite resistivity, let's consider the case where the parameter $\zeta$ (Eq. (67)) is very small (small $\tau_{r}$ ). We assume also that the flow is absent. In this case there appears a possibility of formation of a continuous spectrum of unstable modes even at the current significantly less than the KS current. Indeed, in the limit of a zero $\tau_{r}$, Eq. (67) yields:

$$
\omega^{2}=\mathrm{v}_{A}^{2} \frac{k_{0} k}{2}
$$

Clearly, for the "negative" handedness of the perturbation (such that $k_{0} k<0$ ), perturbations are unstable, with the growth rate

$$
\operatorname{Im} \omega=\mathrm{v}_{A} \sqrt{\frac{\left|k_{0} k\right|}{2}} .
$$

The nature of the mode is as follows. At a high plasma resistivity, the strong axial magnetic field is not frozen into the plasma any more, and a helical perturbation of the appropriate handedness experiences an imbalanced radial force that leads to its growth.

In order to be able to form localized perturbations insensitive to the boundary conditions, we have to assume that $k>>\pi / \mathrm{L}$. Still, the growth rate must be slow enough, so that the condition $\operatorname{Im} \omega \tau_{r}<<1$ holds. These two constraints are compatible with each other provided the condition

$$
\varsigma^{2} \frac{I}{I_{k s}}<<1
$$

is satisfied. In other words, at small enough currents the instability can be present at even relatively high values of $\zeta$ (relatively high conductivity). However, the e-folding time is longer than the resistive time. Therefore, these perturbations will be sensitive to the presence of the plasma flow and will be advected towards the anode. At $\zeta>1$, this requires a relatively low flow velocity,

$$
\mathrm{v}>\frac{\tau_{r} L}{\pi},
$$

i.e. $M>1 / \zeta$.

An interesting feature of the situation with a high plasma resistivity (or low growth rates) is that the column can now slide over the surface of a perfectly conducting anode (with zero sheath resistance). Also interesting can be the fact that, at the parameter $\xi \sim 1$, the perturbation will have a substantial real frequency even if there is no flow.

\section{DISCUSSION}

The phenomenological approach developed in our paper allows one to study the stability of a long-thin plasma column with a number of effects accounted for in a unified manner. Those effects include the presence of the boundaries, axial plasma flow, sheath resistance, and the bulk plasma resistance. We have presented several examples of incorporating some of these effects into an actual analysis.

An important assumption of our model is that there is a boundary beyond which the plasma electrical conductivity essentially vanishes, so that the column is surrounded by a vacuum. We, therefore, will not be able to consider the situations where there is a conducting medium outside the column. One more constraint stems from our assumption that the axial wavelength of the perturbation is much longer than the column radius. 
With these assumptions made, the description of the column deformations becomes quite simple: we characterize them just by a rigid lateral displacements of the plasma slices (Eq. (4)). [With that, we of course, abandon any attempts of describing internal kinks; on the other hand, description of the external kink becomes quite efficient.]

The theory developed in this paper is linear. Strictly speaking, this does not allow us to consider the plasma dynamics at the axial current substantially exceeding the threshold. However, the general structure of the perturbation determined by a linear eigenfunction should be quite a robust entity and thereby give a hint on the shape of the column in the nonlinear regime. A weakly non-linear theory of the type suggested in Ref. 17 , but with the effects of the plasma flow and sheath resistance included, should be feasible.

\section{Acknowledgment}

Work performed for US DOE by UC LLNL under contract \#W-7405-Eng-48. 


\section{References}

1. J.P. Freidberg. Ideal magnetohydrodynamics. Plenum Press, NY, 1987.

2. P.M. Bellan. Phys. Fluids, 12, 058301, 2005.

3. I. Furno, T.P. Intrator, E.W. Hemsing, S.C. Hsu, S. Abbate, P. Ricci, G. Lapenta. Phys. Fluids, 12, 055702, 2005; I. Furno, T. Intrator, E. Torbet, C. Casey, M.D. Cash, J.K. Campbell, W.J. Fienup, C.A. Werley, G.A. Wurden. Rev. Sci. Instr., 74, 2324 (2003).

4. D.D. Ryutov, R.H. Cohen, L.D. Pearlstein. Physics of Plasmas, 11, 4740 (2004).

5. C.C. Hegna. Phys. Plasmas, 11, 4230, 2004.

6. A.W. Hood. Plasma Phys. Contr. Fus., 34, 411 (1992).

7. S.I. Braginski. In: Reviews of Plasma Physics, M.A. Leontovich, Ed., v. 1, p.205, Consultants Bureau, NY, 1965.

8. T.B.Kaiser, W. Nevins, L.D. Pearlstein. Physics of Fluids, 26, 351,1983.

9. M.D. Kruskal, J.L. Tuck. Proc. Roy. Soc. (London), A245, 222 (1958); M.D. Kruskal, J.L. Johnson, M.B. Gottlieb, L.M.Goldman. Phys. Fluids, 1, 421 (1958).

10. V.D. Shafranov, At. Energy, 5, 38 (1956).

11. I.B. Bernstein, E.A. Frieman, M.D. Kruskal, R.M. Kulsrud. Proc. Roy. Soc. A244, $17,1958$.

12. P. Stangeby. The plasma boundary of magnetic fusion devices. IoP Publishing, Bristol, 2000.

13. W. Kunkel, J. Guillory. In: "Phenomena in Ionized Gases" (Proc. 7th Conf. Belgrade, 1965) Vol. 2, p. 702, Belgrade, 1966

14. B.B. Kadomtsev, In: "Phenomena in Ionized Gases" (Proc. 7th Conf. Belgrade, 1965) Vol. 2, p. 610, Belgrade, 1966.

15. D.Farina, R.Pozzoli, D.D. Ryutov. Phys.Fluids, B5, p.4055 (1993).

16. H.L. Berk, D. D. Ryutov, Yu. A.Tsidulko JETP Lett., 52, 23 (1990).

17. I.M. Lanskii and A.I. Shchetnikov. Sov. J. Plasma Phys., 16, 322 (1990). 


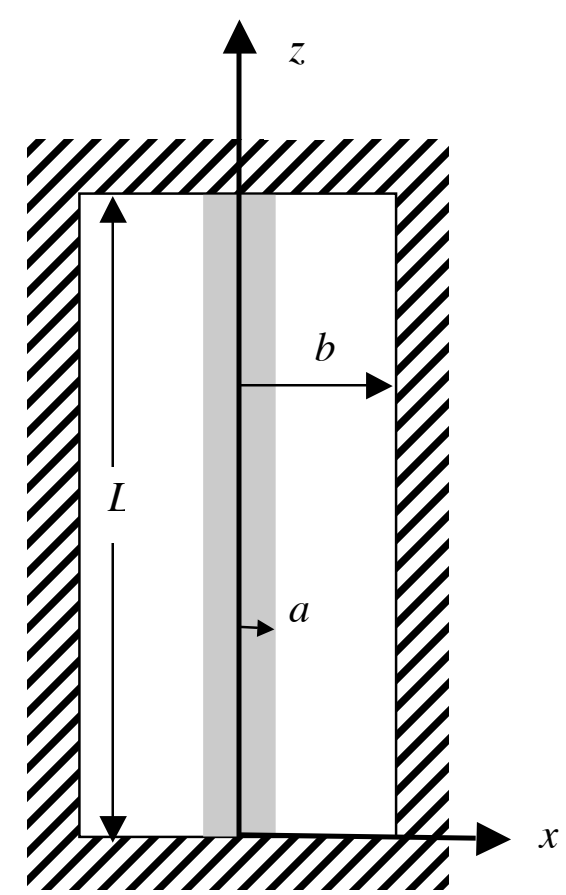

Fig. 1 The geometry of the problem. The axis $y$ is directed away from the viewer. 


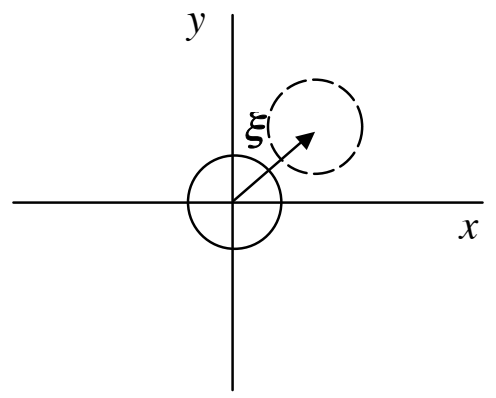

Fig. 2 Deformation of the column. The axis $z$ is directed towards the reader. The solid line represents the initial cross-section, whereas the dashed line shows a displaced cross-section. In each cross-section $z=$ const the displacement vector $\xi$ is the same for all initial points (a rigid displacement).

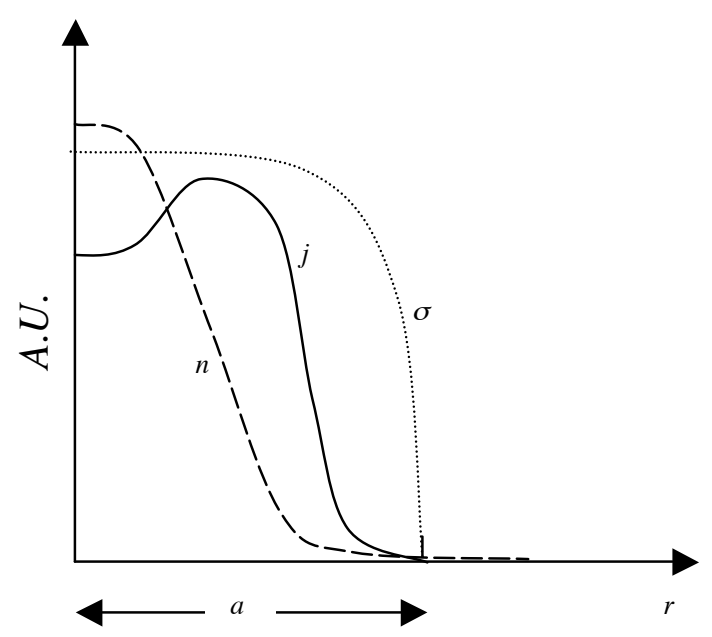

Fig. 3 A sketch of the current density distribution (solid line), the density distribution (dashed line), and the electrical conductivity diatribution (dotted line). As the plasma conductivity depends only of the plasma temperature, the current profile may be broader than the density profile - for a uniform temperature. The current would drop in the zone where the density is so small that the standard model of the plasma conductivity does not work. In the figure, a particular case of a hollow current profile is shown, but our analysis is applicable to an arbitrary distribution. The parameter $a$ that enters our equations is determined by the condition that, at $r<a$, the conductivity is high enough to make the axial magnetic field to be frozen into the plasma. Therefore, $a$ depends on the frequency of perturbations. However, for a steep-enough decrease of the electrical conductivity, $a$ is well-defined. 


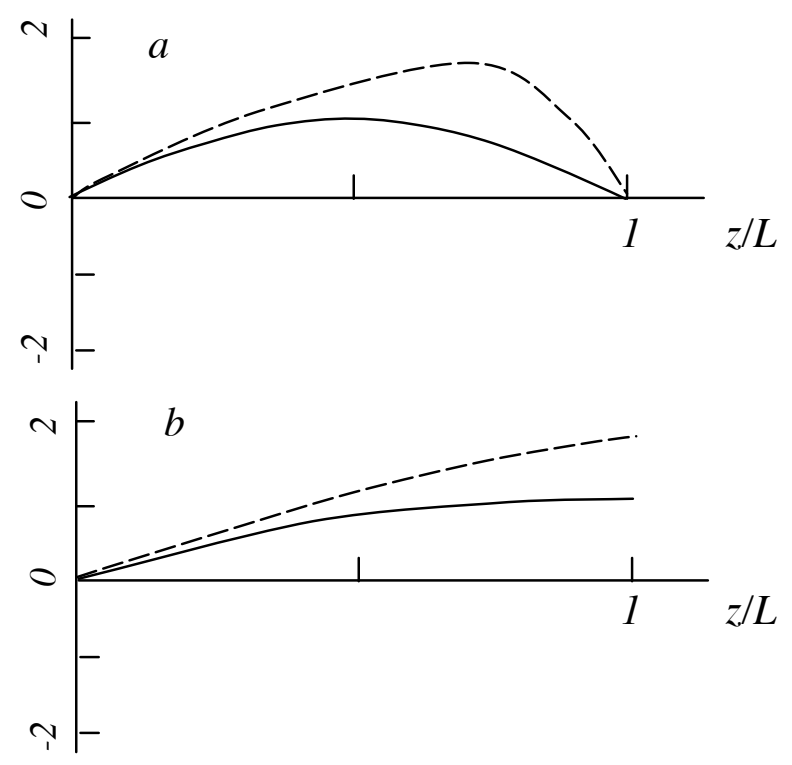

Fig. 4 The shape of the figure of revolution over which the axis of the plasma column is wound: a) Line tying at both $z=0$ and $z=L$; solid curve corresponds to the case of no axial flow; dashed curve corresponds to the axial flow directed from $z=0$ to $z=L$, and to an overcritical situation. b) Line-tying at $z=0$, no line-tying at $z=L$; solid curve corresponds to the case of no flow and to a marginally-stable case in the presence of the flow; dashed curve corresponds to a somewhat supercritical system in the presence of the flow. The flow causes the advection of perturbations and causes a "skewing" of the eigenfunction in the direction of the $z=L$ electrode. 


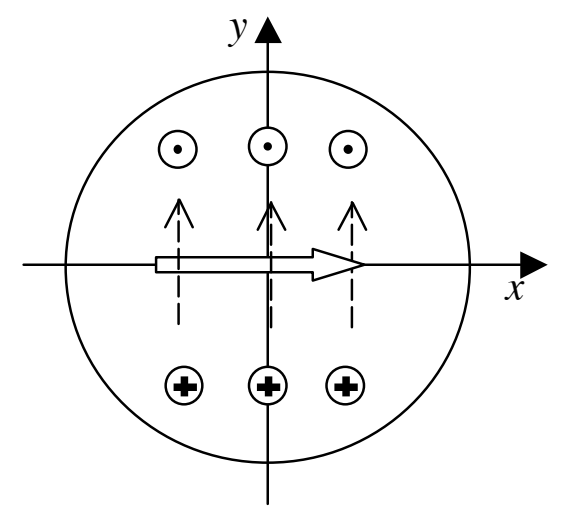

Fig. 5 Sheath reaction to the tilt of the column. The tilt occurs in the $x$ direction, generating the perturbation of the $z$ component of the current; the direction of the current is indicated by the circles with the dots (towards the viewer) and crosses (away from the viewer) inside. The sheath resistance then leads to the formation of electric field on the plasma side of the sheath; the field is directed along the dashed arrows. The presence of the field in the laboratory frame leads to the plasma drift in the direction of the block arrow.

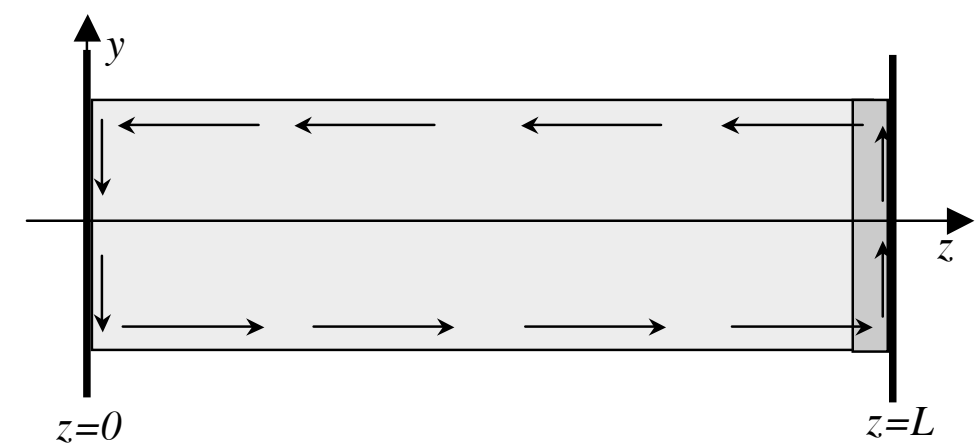

Fig. 6 The current circuit corresponding to the current pattern shown in Fig. 5. The current flows in the opposite directions at the opposite sides of the plasma column (shown in light-gray) and is closed through the plasma sheath (shown in dark-gray) and over the surface of the perfectly conducting electrodes. The only resistive element in this circuit is the sheath. 


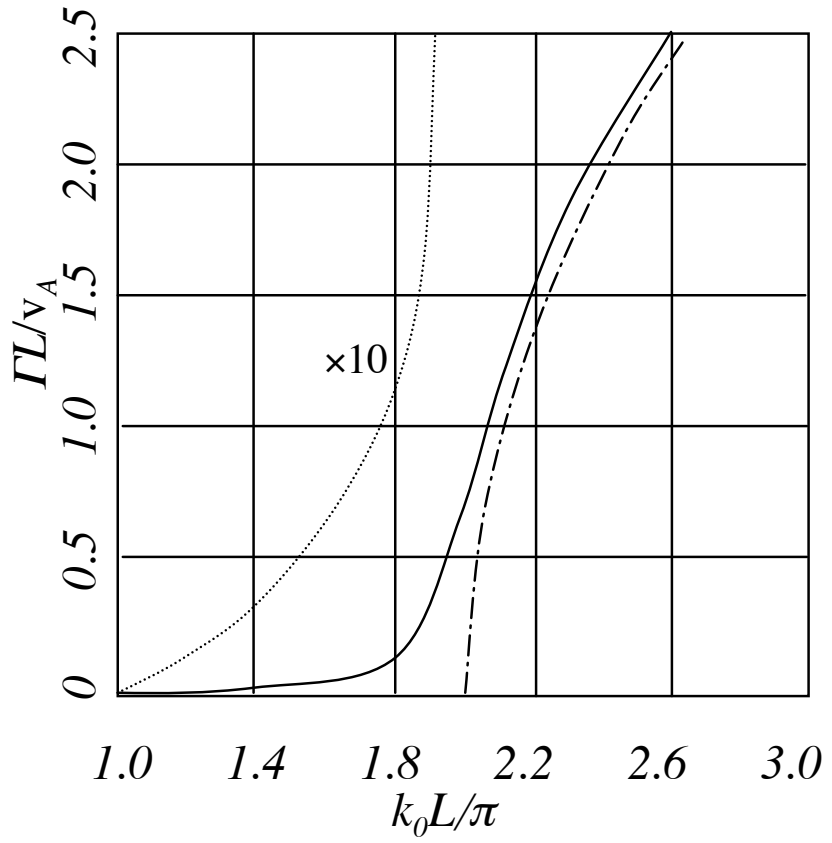

Fig. 7 The growth rate vs the parameter $k_{0}$ for a small value of $\kappa, \kappa=0.02$. This value corresponds to almost complete line-tying, but a weak instability is present even at $k_{0}<2 \pi / L$. The dash-dotted line corresponds to the case of a perfect line-tying, when $\kappa=0$. 